\title{
Ultrafast optical spectroscopy of surface-modified silicon quantum dots: unraveling the underlying mechanism of the ultrabright and color-tunable photoluminescence
}

\author{
Lei Wang ${ }^{1, *}$, Qi Li ${ }^{3, *}$, Hai-Yu Wang ${ }^{1}$, Jing-Chun Huang ${ }^{3}$, Ran Zhang ${ }^{1}$, Qi-Dai Chen ${ }^{1}$, Huai-Liang Xu ${ }^{1}$, Wei Han $^{2}$, \\ Zheng-Zhong Shao ${ }^{3}$ and Hong-Bo Sun ${ }^{1,2}$
}

In this work, the fundamental mechanism of ultrabright fluorescence from surface-modified colloidal silicon quantum dots is investigated in depth using ultrafast spectroscopy. The underlying energy band structure corresponding to such highly efficient direct bandgap-like emissions in our surface-modified silicon quantum dots is unraveled by analyzing the transient optical spectrum, which demonstrates the significant effect of surface molecular engineering. It is observed that special surface modification, which creates novel surface states, is responsible for the different emission wavelengths and the significant improvement in the photoluminescence quantum yields. Following this essential understanding, surface-modified silicon quantum dots with deep blue to orange emission are successfully prepared without changing their sizes.

Light: Science \& Applications (2015) 4, e245; doi:10.1038/Isa.2015.18; published online 30 January 2015

Keywords: quantum confinement; silicon quantum dots; surface molecular engineering; ultrafast spectroscopy; wave function modification

\section{INTRODUCTION}

Crystalline silicon has been the most important semiconductor material in the modern electronics industry due to its excellent electronic properties. However, as a well-known indirect bandgap semiconductor, the optical properties of crystalline silicon are relatively poor, which limits its applications in silicon photonics. To pursue the desired optical performance in silicon materials, nanostructured silicon objects with enhanced photoluminescence (PL) have attracted increasing interest. ${ }^{1-13}$ Most notably, due to the three-dimensional quantum confinement effect in silicon nanocrystals ( $\mathrm{Si} \mathrm{NCs}$ ), the momentum conservation rule is relaxed, and the spatial distributions of photogenerated exciton wave functions tend to extend to the surface of nanoparticles, which provides an efficient approach to manipulate the energy structure of silicon. ${ }^{14-18}$ Thus, the excitonic emission from Si NCs are usually thought to follow three models: (i) 'direct' transition from a quantization-related bandgap; (ii) indirect approaches, i.e., with the help of other emission centers; and (iii) surface and/or strain engineering. ${ }^{19}$

For the 'direct' approach, the observed size-dependent PL behavior in Si NCs can be well explained by the quantum confinement effect. However, such Si NCs can hold strong PL only in the deep red region with limited stability, and their PL lifetimes from these 'direct' transitions are usually on the microsecond time scale. ${ }^{20-22}$ For the indirect approach, recently reported surface-modified Si NCs could also provide highly effective emissive channels. ${ }^{23}$ Furthermore, although the possible modulation mechanisms of the carrier wave function in Si NCs still remain controversial, some researchers have achieved the effect of surface ligands on the low-energy electron states of Si NCs, which would lead to phononless excitonic recombination. ${ }^{24,25}$ Thus, the major drawback of Si NCs could be overcome by these effects solely or synergistically. Nevertheless, insight into the PL mechanism of Si NCs remains elusive, ${ }^{26-31}$ in particular for ultrabright $\mathrm{Si}$ NCs. ${ }^{23,32-34}$ In addition, to further tailor and improve the fluorescent properties of Si NCs, such as their color-tunable fluorescence with high brightness, fundamentally understanding the underlying mechanisms is also required.

Ultrafast spectroscopy is a powerful tool for investigating the electronic structure, excitonic properties and carrier dynamics for various new types of fluorescent nanomaterials, for example, graphene oxide, ${ }^{35,36}$ graphene quantum dots (QDs), ${ }^{37}$ carbon nanodots, ${ }^{38,39}$ polymer nanoparticles, ${ }^{40}$ direct bandgap semiconductor QDs ${ }^{41-43}$ and Si NCs. ${ }^{44-49}$ In our previous work, we produced novel Si NCs with unprecedented ultrabright fluorescence and single-exponential decay. ${ }^{23}$ According to the time-resolved PL dynamics, we demonstrated that no fast PL decay component was present in the range of

${ }^{1}$ State Key Laboratory on Integrated Optoelectronics, College of Electronic Science and Engineering, Jilin University, Changchun 130012, China; ${ }^{2}$ College of Physics, Jilin

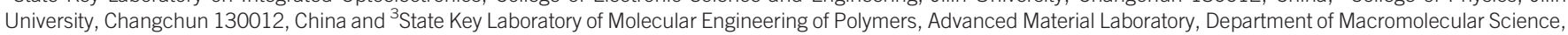
Fudan University, Shanghai 200433, China

*These authors contributed equally to this work

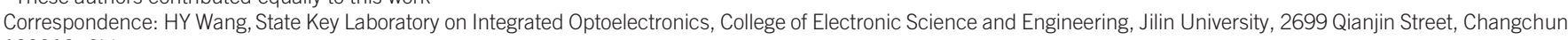
130012, China

E-mail: haiyu_wang@jlu.edu.cn

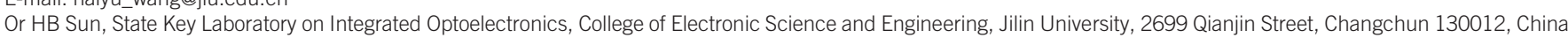
E-mail: hbsun@jlu.edu.cn

Received 16 August 2014; revised 26 November 2014; accepted 1 December 2014; accepted article preview online 4 December 2014 
sub-picosecond to hundreds of nanoseconds in these ultrabright $\mathrm{Si}$ NCs. In addition, their steady-state optical properties, i.e., PL quantum yield (QY), excitation- and temperature-dependent PL behaviors, are closely related to the surface organic agents. Hence, a synergic effect between attached organic ligands and surface oxidized sites was suggested to explain these interesting phenomena; however, a complete understanding of their PL mechanism remains unknown.

Herein, in a quest to explore the fundamental photophysics in these high-quality ultrabright Si NCs, we combined femtosecond transient absorption (TA) spectroscopy with time-resolved fluorescence spectroscopy to systemically investigate the excited-state dynamics in a series of nitrogen-containing organic ligand passivated Si NCs (Figure 1a). After determining what would happen to these photogenerated excitons in the possible excited states, we further unraveled the underlying photophysical model, which was related to the modulation of exciton wave functions due to the surface molecular engineering. We observed that the different emission wavelengths were partly sensitive to their local environment (interaction), i.e., the amount of $\mathrm{N}$-linking phenyl rings, structure of capping agents or linking mode of $\mathrm{N}$ atoms in the ligands anchored to the surface $\mathrm{Si}$ atoms. Si NCs with deep blue to orange emission were then successfully prepared by tailoring their surface instead of changing their sizes. In addition, a novel surface state in the visible range responding to orange-red emission was first revealed using transient absorption spectroscopy of one of the special surface-modified Si NCs.

\section{MATERIALS AND METHODS}

\section{Preparation of Si NCs}

In a typical synthesis of unmodified $\mathrm{Si}$ NCs with surface $\mathrm{Si}-\mathrm{Cl}$ bands (Cl-Si NCs), ${ }^{23} 0.17 \mathrm{~g}$ of sodium and $0.75 \mathrm{~g}$ of naphthalene were stirred in $20 \mathrm{~mL}$ of glyme for approximately $3 \mathrm{~h}$ to produce sodium-naphthalide. Then, $0.20 \mathrm{~mL}$ of $\mathrm{SiCl}_{4}$ was added dropwise. The surface treatment was performed by adding excess capping agents, 0.25-g 1,2,3,4-tetrahydrocarbazole (Te), 0.25-g diphenylamine (Di), 0.25-g carbazole (Ca), 0.25-g 4-methoxydiphenylamine (Me) and $0.25-\mathrm{mL}$ aniline $(\mathrm{An})$ to the as-synthesized Si NCs and stirring the mixture at room temperature for several hours. The solvent and naphthalene were removed under reduced pressure with heating in an oil bath, leaving $\mathrm{Si} \mathrm{NCs}$ with $\mathrm{NaCl}$ and organic byproducts. Finally, water was added to dissolve the Si NCs. Excess organic byproducts and inorganic salts can be removed from the Si NC products by a combination of ultrafiltration and dialysis. The sizes of the resulting Si NCs were in the range of $2-3 \mathrm{~nm}$, with an average value of only approximately $2.5 \mathrm{~nm}$, both before and after surface modification. Transmission electron microscopy images of these products are presented in Supplementary Fig. S1. Because their sizes are much smaller than the exciton Bohr radius of Si crystals (approximately $4.2 \mathrm{~nm}$ ), the products were also so-called surface-modified Si QDs. A high-resolution transmission electron microscopy image of single organically capped QD is also enlarged in each inset of Supplementary Fig. S1. The diamond lattice of silicon can be easily observed, which is further confirmed by the selected-area electron diffraction pattern, powder a

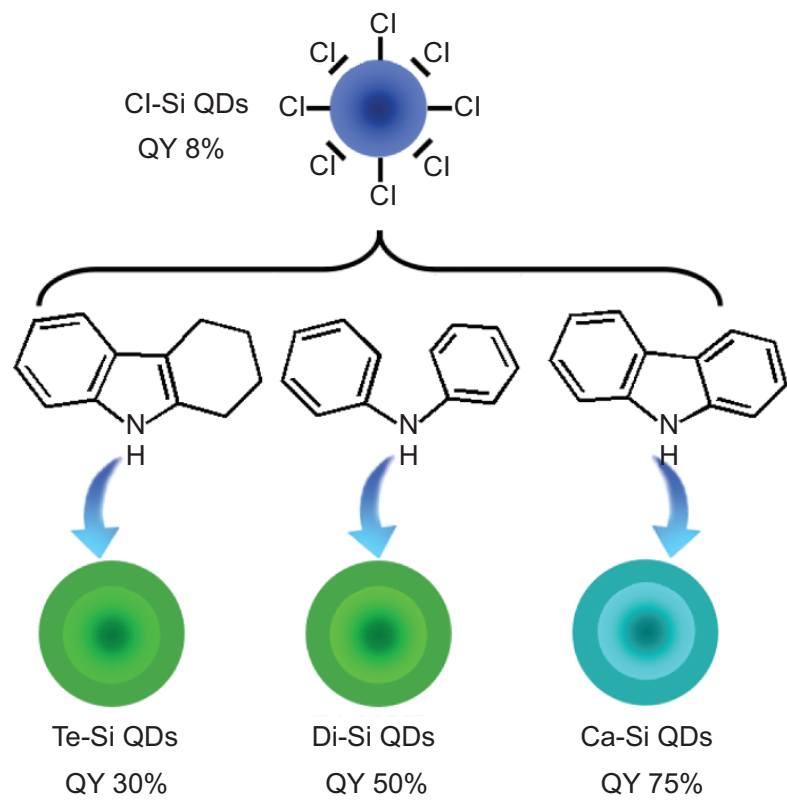

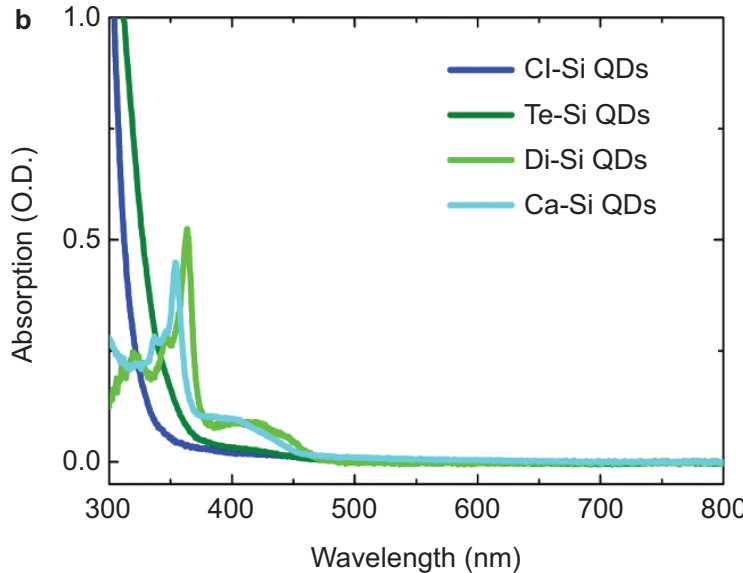

c

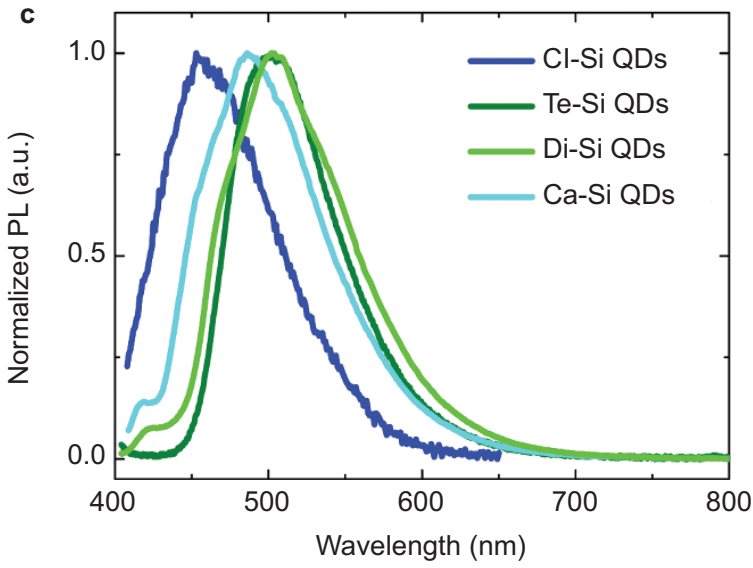

Figure 1 (a) Surface-modified water-dispersible Si QDs with tunable PL. Steady-state absorption (b) and normalized emission spectra (c) for as-synthesized Clterminated and surface-modified Si QDs in water at 400-nm excitation. O.D., optical density; PL, photoluminescence; QD, quantum dot; QY, quantum yield. 
X-ray diffraction pattern and X-ray photoelectron spectroscopy spectrum, as presented in Supplementary Fig. S2.

\section{Femtosecond transient absorption experiments}

In the transient absorption set-up, a mode-locked Ti:sapphire laser/ amplifier system (Solstice; Spectra-Physics, Santa Clara, CA, USA) was used. The output of the amplifier of 1.5-mJ pulse energy, 100-fs pulse width and $250-\mathrm{Hz}$ repetition rate (this low frequency is set for matching the signal collection system) at $800-\mathrm{nm}$ wavelength was split into two parts. The stronger beam was used to generate the 400-nm pump pulses (directly doubled from 800-nm laser pulses), and the other 800 $\mathrm{nm}$ beam was used to generate the broadband white-light probe pulses in the $400-800 \mathrm{~nm}$ range by focusing it into a $2-\mathrm{mm}$-thick water cell. Both the white light and pump light are focused on the sample and overlap with each other in space. The transient absorption data were collected by a fiber-coupled spectrometer connected to a computer. The group velocity dispersion of the transient spectra was compensated by a chirp program. All the samples were stored in a $2-\mathrm{mm}$ cuvette for the TA experiments at room temperature. Pump-power dependent measurements were also performed. In the acceptable range $\left(150-350 \mathrm{~nJ}\right.$ pulse $\mathrm{e}^{-1}$ or $\left.0.5-1.2 \mathrm{~mJ} \mathrm{~cm}^{-2}\right)$, no pump intensity dependent dynamics were observed.

\section{Time-resolved fluorescence experiments}

Subpicosecond time-resolved emission was measured using the femtosecond fluorescence upconversion method. A mode-locked Ti:sapphire laser/amplifier system (Solstice, Spectra-Physics) was again used. The output of the amplifier of 1.5-mJ pulse energy, 100-fs pulse width and $1-\mathrm{kHz}$ repetition rate at $800-\mathrm{nm}$ wavelength was split into two parts; the stronger beam was used to generate excitation light. The resulting fluorescence was collected and focused onto a 1-mm-thick BBO crystal with a cutting angle of $35^{\circ}$. The other part of the regenerative amplifier output was sent into an optical delay line and served as the optical gate for the upconversion of the fluorescence. The generated sum frequency light was then collimated and focused into the entrance slit of a 300-mm monochromator. An ultraviolet-sensitive photomultiplier tube 1P28 (Hamamatsu Photonics, Hamamatsu City, Shizuoka Pref., Japan) was used to detect the signal. The full-width at half-maximum of the instrument response function was approximately 400 fs.

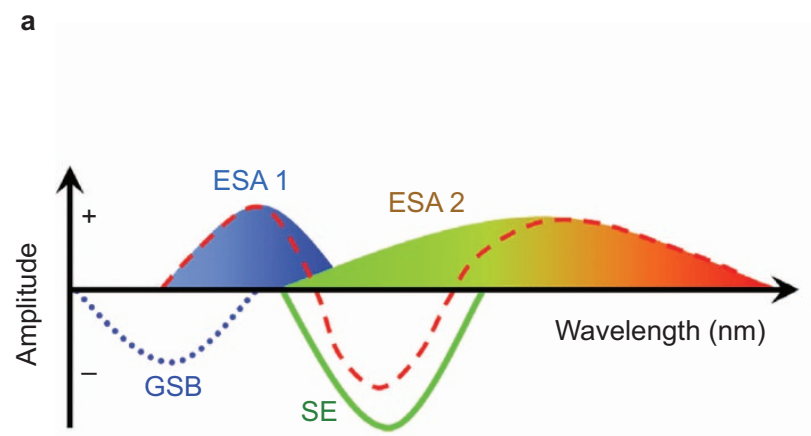

\section{RESULTS AND DISCUSSION}

The steady-state absorption and normalized PL spectra at 400-nm excitation are shown in Figure $1 \mathrm{~b}$ and 1c. For Cl-Si QDs, there is only an absorption tail at approximately $400 \mathrm{~nm}$. After surface modification, the absorption value of the Te-Si QDs increases in this range, whereas the Di-Si QDs and Ca-Si QDs also exhibit distinct absorption peaks. Because no absorption occurs for these organic ligands in the wavelength range beyond $350 \mathrm{~nm}$, this emerged absorption change in the steady-state absorption spectra can be understood as enhanced interaction between ligands and the Si core to some extent. Excited at $360 \mathrm{~nm}(3.44 \mathrm{eV})$, the corresponding PL QY also gradually increases in the sequence of Cl-Si QDs (approximately 5\%), Te-Si QDs (approximately 30\%), Di-Si QDs (approximately 50\%) and Ca-Si QDs (approximately 75\%). The detailed PL QYs measurements are shown in Supplementary Fig. S3. In addition, it is worth noting that additional excitation-dependent PL behavior occurs for the Cl-Si QDs and Te-Si QDs (Supplementary Fig. S4). According to these steady-state results, it appears that the surface capping agents could lead to a different effect on the organically capped Si QDs' band structure, which would dramatically affect the optical properties of the surface-modified Si QDs.

To scrutinize the effect of the surface ligands on the surface-modified Si QDs' band structure, the temporal resolution of the photogenerated carrier dynamics was investigated using femtosecond TA spectroscopy at 400-nm excitation. In general, strong spectral overlapping occurs in the TA spectrum. Even in the relatively simplified TA spectrum, which presented as one possible scenario in Figure 2a, ground-state bleaching, excited-state absorption (ESA) and stimulated emission (SE) occur. Note that only spontaneous emission was experimentally observed when the pump pulse was alone, whereas SE was clearly induced when overlapping the white light probe pulse. However, for the indirect bandgap Si NCs, the presence of a ground-state bleaching signal in the visible range has rarely been reported in previous TA experiments under $400-n m$ excitation. ${ }^{4-49}$ This phenomenon may occur because most reported Si NCs have large Stokes shifts, and their photoluminescence excitation (PLE) peaks are always below $400 \mathrm{~nm}^{16}$ As the PLE peaks of Cl-Si QDs, Te-Si QDs, Di-Si QDs and Ca-Si QDs are also located below $400 \mathrm{~nm}$ (not shown here), which is similar to previously reported results, it is reasonable to ignore the ground-state bleaching signal in the $400-800 \mathrm{~nm}$ probe window and safely assign the negative signals to stimulated emission

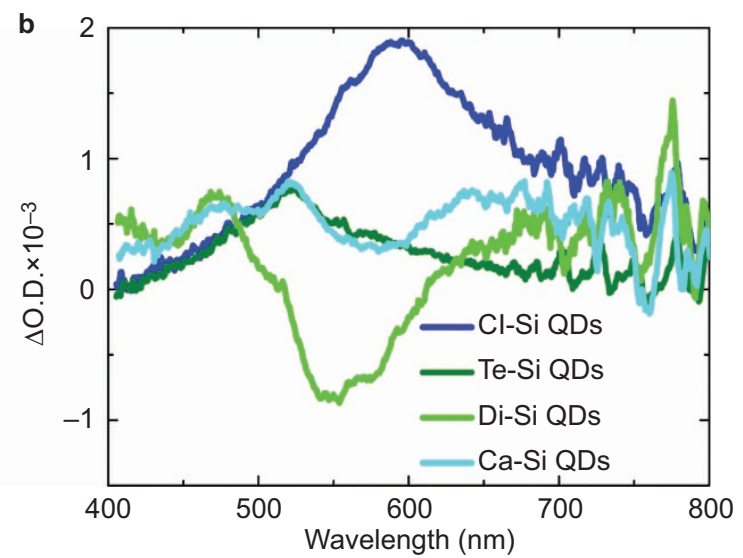

Figure 2 (a) Schematic illustration of global analysis for simplified transient absorption data, ignoring the GSB (blue dot line) signal. The red dashed line represents the TA data obtained experimentally, which can contribute to the overlapping among the two ESA signals and SE signal. (b) Typical transient absorption spectrum (at 1.5 ps) for four Si QDs with different surface modifications at 400-nm excitation. ESA, excited-state absorption; GSB, ground-state bleaching; O.D., optical density; QD, quantum dot; SE, stimulated emission; TA, transient absorption. 
for the four samples in the following analysis. Thus, there are still two signals, ESA and SE, overlapping with each other. Fortunately, if we can obtain the PL lifetime (equal to the lifetime of the SE component corresponding to the pure radiative recombination) using other approaches, such as time-correlated single-photon counting or femtosecond time-resolved PL experiments, these signals (ESA and SE) with the same lifetime components but different amplitudes at each wavelength can be reasonably separated according to a global analysis.

In Figure 2b, the typical TA spectra (at 1.5 ps) for the as-synthesized Cl-terminated Cl-Si QDs and surface-modified Si QDs with different surface capping agents are displayed (presented as the entire transient spectral evolution for each Si QD sample in Supplementary Fig. S5). These apparent differences in the TA spectra imply that the underlying excited-state processes in each surface-modified Si QD sample are indeed largely affected by the surface ligands. The corresponding global analysis for the TA data of surface-modified Si QDs on the time scale from 0.8 to 1330 ps is presented in Figure 3. As mentioned before, one of the lifetime components is fixed and assigned to the radiative recombination process according to the reported average PL lifetimes, that is, 4, 15, 17 and $31 \mathrm{~ns}$ for Cl-Si QDs, Te-Si QDs, Di-Si QDs and Ca-Si QDs, respectively. ${ }^{23}$ These long-lifetime fluorescent states containing stimulated emission (negative signal) accompanied with the corresponding excited-state absorption (positive signal) are well identified in the following global fitting results.

For Cl-Si QDs (Figure 3a), it is apparent that there are two lifetime components related to emission (negative signals). One component is the fixed lifetime component of $4 \mathrm{~ns}$, contributing to blue fluorescence; the other component is a very short-lifetime component of only $1.4 \mathrm{ps}$, which is mainly attributed to green emission with very low PL QY due to the fast decay. Thus, the remaining lifetime component of 21 ps can be safely assigned to nonradiative recombination. Notably, this type of the excited-state behavior is also suitable for Te-Si QDs (Figure 3b), except that the long-lifetime PL component of $15 \mathrm{~ns}$ corresponds to green emission, whereas the short-lifetime component of 1.0 ps corresponds to blue emission. Hence, these global fitting results are consistent with the previously observed excitation-dependent PL behaviors. Such experimental results clearly demonstrate the intrinsic correlation between TA spectra and PL data, which provides an exciting step toward obtaining a comprehensive understanding of the photophysical properties in our surface-modified colloidal Si QDs. Moreover, our experiment results also directly demonstrate that although the surface ligands are completely different for Cl-Si QDs and Te-Si QDs, their energy band structures are similar. The only difference is the emission band, which can be effectively modulated by surface chemical modification.

The question subsequently becomes how to explain the ultrabright fluorescence from Di-Si QDs and Ca-Si QDs. Fortunately, the ultrafast spectroscopic analysis on the two samples can provide a crucial clue in answering these questions. Figure $3 \mathrm{c}$ and $3 \mathrm{~d}$ presents the global analysis for the TA spectra of the Di-Si QDs and Ca-Si QDs, which indicates that the spectral structures in the TA spectra for the latter two types of surface-modified Si QDs mainly originated from the radiative recombination states with lifetime components of $17 \mathrm{~ns}$ and $31 \mathrm{~ns}$, respectively. In addition, there are three remaining lifetime
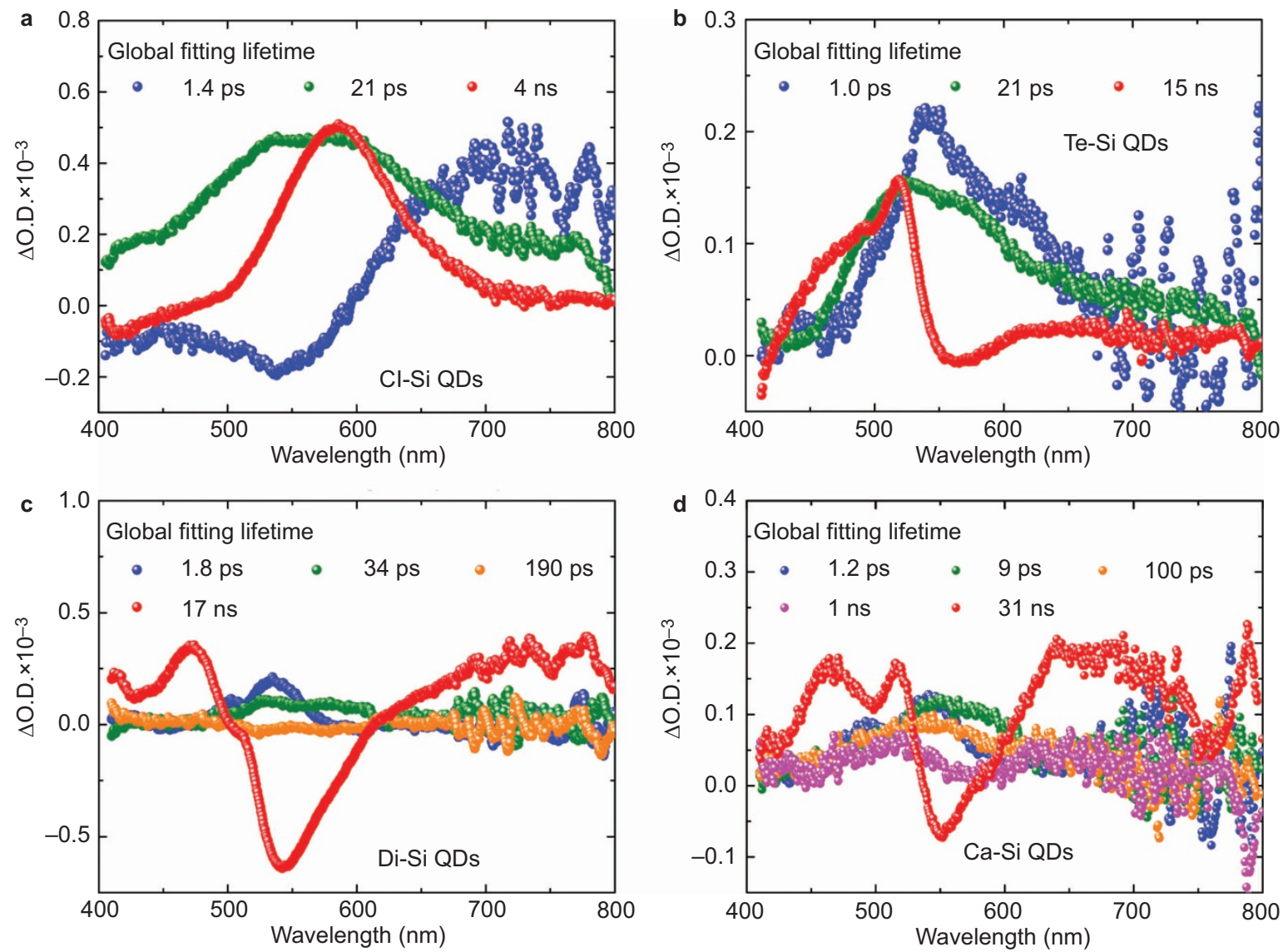

Figure 3 Global analysis for the transient absorption data of (a) Cl-Si QDs, (b) Te-Si QDs, (c) Di-Si QDs and (d) Ca-Si QDs. O.D., optical density; QD, quantum dot. 
components (1.8 ps, 34 ps and 190 ps) for Di-Si QDs and four lifetime components (1.2 ps, 9 ps, 100 ps and $1 \mathrm{~ns}$ ) for Ca-Si QDs, which lack an obvious spectral structure and thus are most likely related to nonradiative excited-state processes.

Together with Te-Si QDs, the three types of surface-modified Si QDs with cyan-green emission and single-exponential decay possess almost the same size distributions and extremely similar surface agents, as presented in Figure 1a. Compared with the surface ligands in Ca-Si QDs, there is only a lack of a chemical bond linking the two phenyl rings for Di-Si QDs and an increase of four hydrogen atoms in one phenyl ring for Te-Si QDs. Therefore, it appears that surfacemodified Si QDs samples with higher PL QY exhibit longer and more complex excited-state behaviors. The characterization dynamics probed at the same wavelength $(500 \mathrm{~nm})$ extracted from femtosecond time-resolved PL dynamics and TA spectra under 400-nm excitation for Di-Si QDs and Ca-Si QDs are presented in Supplementary Fig. S6, which clearly demonstrates that with the decay of TA signals, the PL dynamics almost remain constant on the same time scale, which can be indicated by a single-exponential PL lifetime. However, such a long PL lifetime (17 ns for Di-Si QDs and $31 \mathrm{~ns}$ for Ca-Si QDs) leads to a very low signal-to-noise ratio in the femtosecond time-resolved PL experiments.

This finding indicates that the complex excited-state processes observed in TA analysis are completely irrelevant to the pure exciton radiative recombination obtained in time-resolved PL experiments. In other words, these results unambiguously demonstrate that these multi-exponential excited-state lifetime components arise from nonradiative recombination processes, i.e., intra-band hot-carrier relaxation. In general, higher temperature will lead to faster hot carrier relaxation, which could shorten the PL lifetime and consequently decrease the steady-state PL intensity, similar to the case of Di-Si QDs. ${ }^{23}$ However, due to the intrinsic slower hot-carrier relaxation in $\mathrm{Ca}-\mathrm{Si} \mathrm{QDs}$, this temperature effect could be greatly reduced. Therefore, the temperature-dependent PL behavior for Ca-Si QDs is not obvious compared with Di-Si QDs. These temperature-dependent steady-state PL spectra are presented in Supplementary Fig. S7. In addition, it is worth noting that these nonradiative recombination processes in Di-Si QDs and Ca-Si QDs are also different from those in $\mathrm{Cl}-\mathrm{Si}$ QDs and Te-Si QDs because these processes are irrelevant to any spectral evolution. Hence, there are indeed two types of PL mechanisms.

As stated above, due to the quantum confinement effect, larger amplitudes of carrier wave functions in Si QDs are located at the surface of nanoparticles. Thus, the effect of the surface ligands on the Si QDs' band structure (that is, the core-ligand interaction) must be considered. We suggest that this wave function modification by surface modification is common, whether for traditional $\mathrm{O}-, \mathrm{H}$ - and C-terminated Si NCs or, in our case, Cl-terminated Si QDs and Ncontaining organic ligands modified Si QDs; however, the effect on the excited-state processes is different. The possible energy diagram for the two types of surface-modified Si QDs at the lowest energy levels modified by surface ligands is presented in Figure 4.

For a moderate effect of the surface ligands, namely, the 'weak' interaction mode, this modification only creates some direct transition energy levels within the intrinsic indirect bandgap (Figure 4, left panel). For this type of surface-modified Si QDs, there is a remarkable sign, that is, a fast spectral evolution in transient absorption spectra accompanied with no special absorption structure in steady-state absorption spectra. However, for the cases where the excited-state processes of surface-modified Si QDs can be strongly affected by the

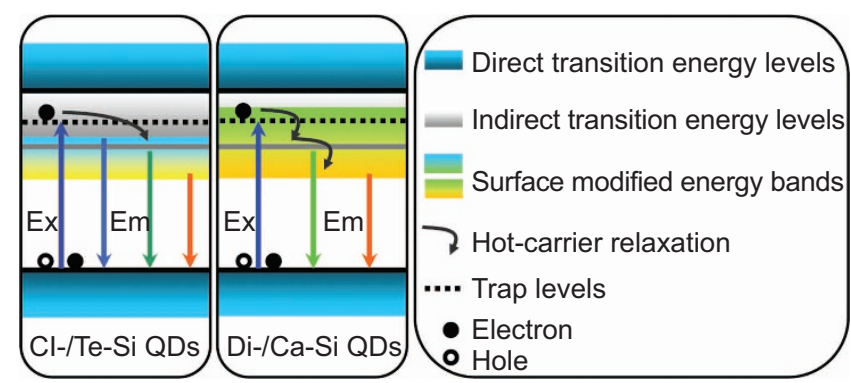

Figure 4 Schematic illustration of the energy level arrangement for the two types of surface-modified Si QDs. Em, emission; Ex, excitation; QD, quantum dot.

surface ligands, namely, the 'strong' interaction mode, the intrinsic indirect bandgap of the Si QDs is almost filled with surface-modified energy bands, which becomes an integrated direct transition energy structure (Figure 4, middle panel). Therefore, there is a strong stimulated emission signal and no spectral evolution in transient absorption spectra. In addition, special absorption structures in the band edge can be easily observed in their steady-state absorption spectra. Recently, Kůsová et al. ${ }^{50}$ reported the presence of tensile strain in organic passivated Si NCs. This effect could also exist in our studied surface-modified Si QDs, i.e., surface strain could also be one part of the so-called core-ligand interaction.

After obtaining the energy band structure model of the surfacemodified Si QDs, the next issue is to further illustrate the nature of such a dominant integrated band, which is the origin of ultrabright fluorescence. Namely, is this phenomenon still a modified core-related state or a novel surface state? To this end, we performed some additional experiments on the ultrabright surface-modified Si QDs that were relevant to surface oxidation and the solvent effect. As previously mentioned, ${ }^{23}$ in addition to the crucial role of organic ligands, surface oxidation is also necessary to achieve such ultrabright fluorescence in Di-Si QDs and Ca-Si QDs. For example, we have investigated the detailed evolution of Di-Si QDs' fluorescence under various oxidation degrees (Supplementary Fig. S8).

We observed that when Di-Si QDs were initially dissolved in water with a low degree of oxidation, only a moderate blue photoluminescence was observed. After $100 \mathrm{~h}$ of storage, the ultrabright green fluorescence gradually occurred, and a higher degree of oxidation could be observed in the Fourier transform infrared spectroscopy measurement. A controlled experiment was also conducted in which all the oxygen during storage was removed. In this case, no ultrabright green fluorescence was observed. In general, two types of fluorescence changes can be observed when silicon nanoparticles are oxidized. If the PL appears blueshifted, this PL is often regarded as core-emission related because during the oxidation progresses, the size of the silicon core will be reduced such that its PL band gap will increase based on the quantum confinement effect. ${ }^{51}$ However, some other researchers hold a different viewpoint that such a change of steady-state PL in Si QDs is caused by a blue emission surface center induced during the oxidation process. ${ }^{52}$ In our sample, a new red-shifted PL occurred during oxidation. A possible explanation for this phenomenon is that new surface states constitute during oxidation..$^{15,18,24}$

For the solvent effect of the fluorescence from our surface-modified Si QDs, the steady-state PL in the surface-modified Si QDs exhibits a more or less pronounced blueshift accompanied by the reduction of the PL lifetime when the solvent is changed from water to acetone (examples are presented in Supplementary Tables S1 and S2). These results are consistent with recently reported data on the oxidation of 
$\mathrm{N}$-containing Si NCs, where their photoluminescence was demonstrated to originate from the surface $\mathrm{SiO}_{\mathrm{x}} \mathrm{N}_{\mathrm{y}}$ charge transfer state. ${ }^{53}$ All these experimental results suggest a novel surface state as the dominant origin of the ultrabright fluorescence. Both organic ligands and surface oxidation are necessary to form this state, and the structure of ligands is crucial in determining whether such a state can dominate the entire emission process. Herein, it should be noted that rigorous theoretical modeling is needed to fully elucidate the effect of a particular surface capping on the Si QDs' band structure, which will be performed in the future.

Because the organic ligands containing $\mathrm{N}$-linking phenyl rings have exhibited great potential for modulating the energy structure of surface-modified Si QDs, we further use other simple N-containing organic ligands-4-methoxydiphenylamine and aniline to modify $\mathrm{Cl}-$ Si QDs. Noting that there are two N-H bonds in aniline, both of which could be expected to react with the $\mathrm{Si}-\mathrm{Cl}$ bonds on the surface of $\mathrm{Si}$ QDs, the resulting steady-state absorption spectrum for aniline-modified Si QDs (An-Si QDs) exhibits a new absorption structure in the range of 450-550 $\mathrm{nm}$ (Figure 5a). Surprisingly, the corresponding PL spectra excited in this range exhibit a bright orange fluorescence (Figure 5b). In contrast, for 4-methoxydiphenylamine modified $\mathrm{Si}$ QDs (Me-Si QDs, Figure 5c), such a slight change in the surface ligand in comparison with diphenylamine leads to a remarkable blueshift of emission (Figure 5d).

The photoluminescence excitation spectrum corresponding to the orange emission in the An-Si QDs peaks at approximately $500 \mathrm{~nm}$
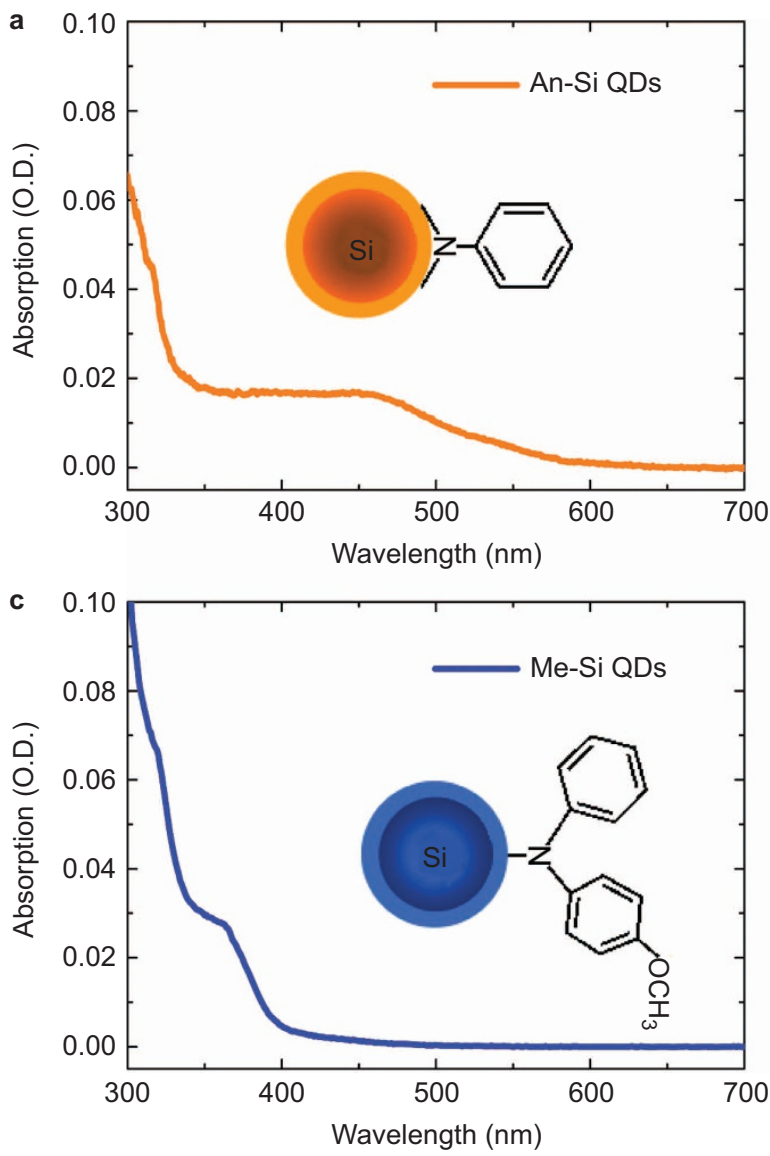

(Figure 6, top panel). This result is interesting because as we mentioned before, for most previously reported Si QDs, the PLE peaks are located in the ultraviolet range because of the very low density of states near their band edge. To obtain deeper insight into this phenomenon, we performed a femtosecond transient absorption experiment and PL dynamics measurement using the time-correlated single-photon counting technique for An-Si QDs under 500-nm excitation (Supplementary Fig. S9). Interestingly, when this emission species is excited, there is a large negative signal in the range of approximately $470-650 \mathrm{~nm}$ in the TA spectra, which presents a fast decay followed by a long lifetime component. In contrast, only a 4.5-ns PL lifetime was obtained, which was probed at $600 \mathrm{~nm}$ and represents the pure radiative recombination process. Fixing one of the lifetime components at 4.5 ns (denoting its PL lifetime) in the global analysis of the An-Si QDs' TA data (Figure 6, bottom panel), we observed that in the obtained five lifetime components, two ( $88 \mathrm{ps}$ and $4.5 \mathrm{~ns}$ ) exhibited negative signals centered at approximately $500 \mathrm{~nm}$. This finding corresponds with the emission species observed in the PLE spectrum. Therefore, these lifetime components of 88 ps and 4.5 ns are the ground-state bleaching signals of the special emission species. For the other lifetime components (1.1 ps, $6.8 \mathrm{ps}$ and $370 \mathrm{ps}$ ), their negative signals cover the entire PL emission range of An-Si QDs at 500-nm excitation. Thus, these signals are attributed to the stimulated emission.

Note that with the help of the PLE spectrum and time-resolved PL dynamics, analyzing the TA data using global analysis in this case remains reasonable. At least, this analysis cannot change the intrinsic
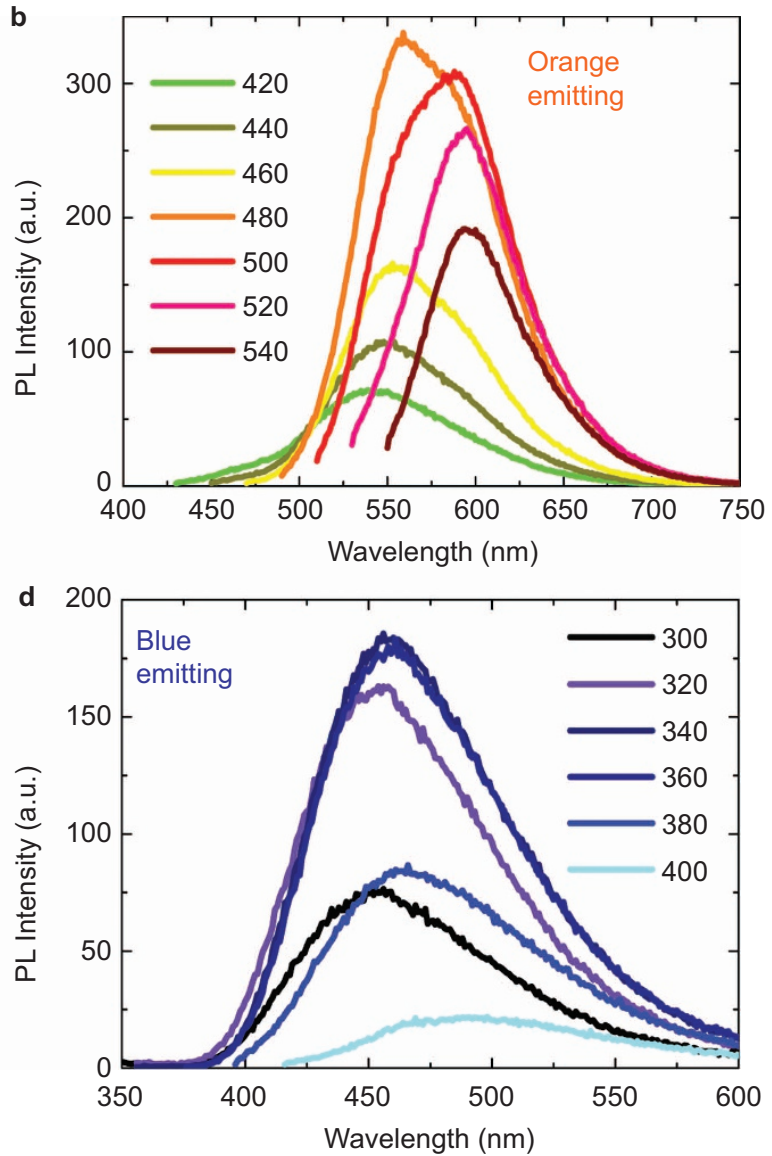

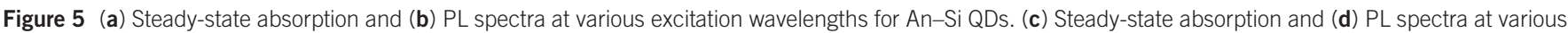
excitation wavelengths for Me-Si QDs. O.D., optical density; QD, quantum dot. 


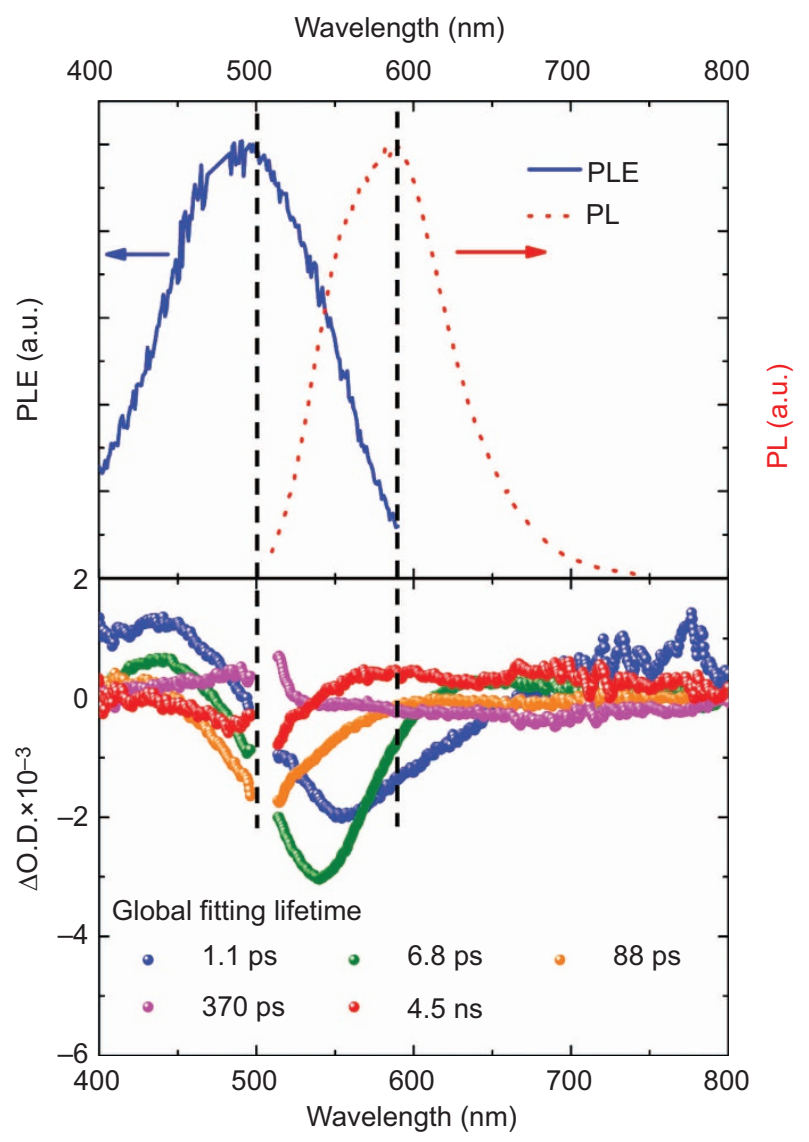

Figure 6 (Top panel) Photoluminescence excitation spectrum (blue solid line) corresponding to orange emission (probed at $600 \mathrm{~nm}$ ) and steady-state emission spectrum (red dot line) under 500-nm excitation in An-Si QDs. (Bottom panel) Global analysis for the transient absorption data of An-Si QDs under 500-nm excitation; the vertical dashed lines indicate the positions corresponding to the PLE peak (approximately $500 \mathrm{~nm}$ ) and PL peak (approximately $590 \mathrm{~nm}$ ) in the top panel. O.D., optical density; PL, photoluminescence; PLE, photoluminescence excitation; $\mathrm{QD}$, quantum dot.

feature of each lifetime component. Hence, in accordance with our proposed model, this TA experiment unambiguously demonstrates that the observed emission state in the visible range originates from the surface modification. Moreover, the results also suggest that these lifetime components, except for the ground-state bleaching component of 4.5 ns in An-Si QDs, are nonradiative, which is consistent with the recent results reported by Hannah et al. ${ }^{54}$ It appears that there are some common PL properties between carbon nanodots and surfacemodified Si NCs from the surface emission state viewpoint. These photophysical studies on organically capped Si NCs may open an intriguing window to obtain tunable PL in carbon nanodots and graphene QDs.

According to our criteria stated above, both the An- and Me-Si QDs could belong to the 'weak' interaction mode; however, following our proposed technical approach, deep blue, cyan-green, and orange emissions from surface-modified Si QDs were achieved without changing their sizes. Recently, Dasog et al. ${ }^{55}$ also reported Si NCs with tunable PL across the visible spectrum via rational variation in the surface moieties. Thus, our next goal is to further improve the PL QY of the surface-modified Si QDs with orange-red emission ('strong' interaction mode) and illustrate the relationship between the organic agent structure and Si QDs' emission color in more depth using other methods such as quantum calculations.

\section{CONCLUSIONS}

In summary, our ultrafast spectroscopy study clearly demonstrates the relationship between surface molecular engineering and the energy band structure of Si QDs. The correlation between the excited-state processes in transient spectra and the steady-state PL properties (excitation-wavelength dependent and temperature-dependent PL behaviors) is also fully discussed. The underlying PL mechanisms are unraveled in detail, that is, there are two types of interaction modes for surface-modified Si QDs: 'weak' interaction and 'strong' interaction, relying on the effect of surface ligands on the excited-state processes (interaction strength of the core-ligand interaction) in $\mathrm{Si}$ QDs. The former usually includes as-synthesized Cl-Si QDs with weak blue emission and moderate fluorescence-emitting $\mathrm{Me}-$, $\mathrm{Te}-$ and $\mathrm{An}-$ Si QDs, in which the emission wavelengths can be tuned by different surface modification. These emissions are simply common moleculelike emissions. For the latter, in the preparation of surface-modified Si NCs, by specially designing the molecular structure of organically capping agents to increase the density of state on the surface of $\mathrm{Si}$ NCs (i.e., more phenyl rings in the surface ligands) and further restricting the molecular vibrational/torsional motion, the ultrabright photoluminescence can emerge, as for the cases of Di- and Ca-Si QDs, in which the surface oxidation most likely also plays an important role. This finding is attributed to the special integrated direct transition energy structure, where the core-ligand interaction is so strong that the entire intrinsic indirect bandgap of the Si QDs is almost filled by the surface-modified energy bands. In this manner, these core-ligand interactions could also be useful for the enhancement of fluorescent emission in other Si nanostructures, for example, two-dimensional Si nanosheets. ${ }^{56}$ Finally, depending on the modification of exciton wave functions by the core-ligand interaction, tunable emissions from deep blue to orange can be successfully obtained from surface chemically modified Si QDs without changing their sizes.

\section{ACKNOWLEDGEMENTS}

HBS thanks the Natural Science Foundation of China (NSFC) under Grant Nos. 91423102, 21273096, 91323301 and 11104109, and the National Basic Research Program of China (973 Program) under Grant No. 2014 CB921302 for support.

1 Pavesi L, Dal Negro L, Mazzoleni C, Franzò G, Priolo F. Optical gain in silicon nanocrystals. Nature 2000; 408: 440-444.

2 Ding ZF, Quinn BM, Haram SK, Pell LE, Korgel BA et al. Electrochemistry and electrogenerated chemiluminescence from silicon nanocrystal quantum dots. Science 2002; 296: 1293-1297.

3 Cloutier SG, Kossyrev PA, Xu J. Optical gain and stimulated emission in periodic nanopatterned crystalline silicon. Nat Mater 2005; 4: 887-891.

4 Liu CY, Holman ZC, Kortshagen UR. Optimization of Si NC/P3HT hybrid solar cells. Adv Funct Mater 2010; 20: 2157-2164.

5 He Y, Su YY, Yang XB, Kang ZH, Xu TT et al. Photo and pH stable, highly-luminescent silicon nanospheres and their bioconjugates for immunofluorescent cell imaging. J Am Chem Soc 2009; 131: 4434-4438.

6 He Y, Zhong YL, Peng F, Wei XP, Su YY et al. One-pot microwave synthesis of waterdispersible, ultraphoto- and $\mathrm{pH}$-stable, and highly fluorescent silicon quantum dots. J Am Chem Soc 2011; 133: 14192-14195.

7 Erogbogbo F, Yong KT, Roy I, Hu R, Law WC et al. In vivo Targeted cancer imaging, sentinel lymph node mapping and biocompatible silicon nanocrystals. ACS Nano 2011; 5: 413-423.

8 Puzzo DP, Henderson EJ, Helander MG, Wang ZB, Ozin GA et al. Visible colloidal nanocrystal silicon light-emitting diode. Nano Lett 2011; 11: 1585-1590.

9 Wolf O, Dasog M, Yang Z, Balberg I, Veinot JG et al. Doping and quantum confinement effects in single Si nanocrystals observed by scanning tunneling spectroscopy. Nano Lett 2013; 13: 2516-2521. 
10 Vach $\mathrm{H}$. Ultrastable silicon nanocrystals due to electron delocalization. Nano Lett 2011; 11: 5477-5481.

11 Wheeler LM, Neale NR, Chen T, Kortshagen UR. Hypervalent surface interactions for colloidal stability and doping of silicon nanocrystals. Nat Commun 2013; 4: 2197.

12 Zhang YX, Han X, Zhang JM, Liu Y, Huang $\mathrm{H}$ et al. Photoluminescence of silicon quantum dots in nanospheres. Nanoscale 2012; 4: 7760-7765.

13 Kang ZH, Liu Y, Lee ST. Small-sized silicon nanoparticles: new nanolights and nanocatalysts. Nanoscale 2011; 3: 777-791.

14 Hybertsen MS. Absorption and emission of light in nanoscale silicon structures. Phys Rev Lett 1994; 72: 1514-1517.

15 Wolkin MV, Jorne J, Fauchet PM, Allan G, Delerue C. Electronic states and luminescence in porous silicon quantum dots: the role of oxygen. Phys Rev Lett 1999; 82: 197-200.

16 Hessel CM, Reid D, Panthani MG, Rasch MR, Goodfellow BW et al. Synthesis of ligandstabilized silicon nanocrystals with size-dependent photoluminescence spanning visible to near-infrared wavelengths. Chem Mater 2012; 24: 393-401.

17 de Boer WD, Timmerman D, Dohnalová K, Yassievich IN, Zhang H et al. Red spectral shift and enhanced quantum efficiency in phonon-free photoluminescence from silicon nanocrystals. Nat Nanotechnol 2010; 5: 878-884.

18 Shirahata N. Colloidal Si nanocrystals: a controlled organic-inorganic interface and its implications of color-tuning and chemical design toward sophisticated architectures. Phys Chem Chem Phys 2011; 13: 7284-7294.

19 Priolo F, Gregorkiewicz T, Galli M, Krauss TF. Silicon nanostructures for photonics and photovoltaics. Nat Nanotechnol 2014; 9: 19-32.

20 Wilson WL, Szajowski PF, Brus LE. Quantum confinement in size-selected, surfaceoxidized silicon nanocrystals. Science 1993; 262: 1242-1244.

21 Meier C, Gondorf A, Lüttjohann S, Lorke A, Wiggers H. Silicon nanoparticles: absorption, emission, and the nature of the electronic bandgap. J App/ Phys 2007; 101: 103112

22 Miller JB, Van Sickle AR, Anthony RJ, Kroll DM, Kortshagen UR et al. Ensemble brightening and enhanced quantum yield in size-purified silicon nanocrystals. ACS Nano 2012; 6: 7389-7396.

$23 \mathrm{Li} \mathrm{Q}, \mathrm{He} \mathrm{Y}$, Chang J, Wang L, Chen HZ et al. Surface-modified silicon nanoparticles with ultrabright photoluminescence and single-exponential decay for nanoscale fluorescence lifetime imaging of temperature. J Am Chem Soc 2013; 135: 14924-14927.

24 Dohnalová K, Poddubny AN, Prokofiev AA, de Boer WD, Umesh CP et al. Surface brightens up Si quantum dots: direct bandgap-like size-tunable emission. Light Sci App/2013; 2: e47; doi:10.1038/lsa.2013.3.

25 Dohnalová K, Fučíková A, Umesh CP, Humpolíčková J, Paulusse JM et al. Microscopic origin of the fast blue-green luminescence of chemically synthesized non-oxidized silicon quantum dots. Small 2012; 8: 3185-3191.

26 Godefroo S, Hayne M, Jivanescu M, Stesmans A, Zacharias M et al. Classification and control of the origin of photoluminescence from Si nanocrystals. Nat Nanotechnol 2008; 3: 174-178.

27 Kůsová K, Cibulka O, Dohnalová K, Pelant I, Valenta J et al. Brightly luminescent organically capped silicon nanocrystals fabricated at room temperature and atmospheric pressure. ACS Nano 2010; 4: 4495-4504.

28 Anthony R, Kortshagen U. Photoluminescence quantum yields of amorphous and crystalline silicon nanoparticles. Phys Rev B 2009; 80: 115407.

$29 \mathrm{Hao} \mathrm{HL}$, Shen WZ. Identification and control of the origin of photoluminescence from silicon quantum dots. Nanotechnology 2008; 19: 455704

30 Sa'ar A. Photoluminescence from silicon nanostructures: the mutual role of quantum confinement and surface chemistry. J Nanophotonics 2009; 3: 032501.

31 Sa'ar A. On the origin of photoluminescence from silicon nanostructures: a new perspective. Phys Status Solidi C 2011; 8: 1764-1768.

32 Belomoin G, Therrien J, Smith A, Rao S, Twesten R et al. Observation of a magic discrete family of ultrabright Si nanoparticles. Appl Phys Lett 2002; 80: 841-843.

33 Jurbergs D, Rogojina E, Mangolini L, Kortshagen U. Silicon nanocrystals with ensemble quantum yields exceeding 60\%. Appl Phys Lett 2006; 88: 233116.

34 Hannah DC, Yang JH, Podsiadlo P, Chan MK, Demoritière A et al. On the origin of photoluminescence in silicon nanocrystals: pressure-dependent structural and optical studies. Nano Lett 2012; 12: 4200-4205.

35 Wang L, Wang HY, Wang Y, Zhu SJ, Zhang YL et al. Direct observation of quantumconfined graphene-like states and novel hybrid states in graphene oxide by transient spectroscopy. Adv Mater 2013; 25: 6539-6545.

36 Zhang Q, Zheng HJ, Geng ZG, Jiang SL, Ge J et al. The realistic domain structure of assynthesized graphene oxide from ultrafast spectroscopy. J Am Chem Soc 2013; 135: 12468-12474.
37 Wang L, Zhu SJ, Wang HY, Wang YF, Hao YW et al. Unraveling bright molecule-like state and dark intrinsic state in green-fluorescence graphene quantum dots via ultrafast spectroscopy. Adv Opt Mater 2013; 1: 264-271.

38 Wen XM, Yu P, Toh YR, Hao XT, Tang J. Intrinsic and extrinsic fluorescence in carbon nanodots: ultrafast time-resolved fluorescence and carrier dynamics. Adv Opt Mater 2013; 1 : 173-178.

39 Wang L, Zhu SJ, Wang HY, Qu SN, Zhang YL et al. Common origin of green luminescence in carbon nanodots and graphene quantum dots. ACS Nano 2014; 8 : 2541-2547.

40 Wang L, Wu CF, Wang HY, Wang YF, Chen QD et al. Internal structure-mediated ultrafast energy transfer in self-assembled polymer-blend dots. Nanoscale 2013; 5: 7265-7270.

41 Wang L, Wang HY, Gao BR, Pan LY, Jiang Y et al. Transient absorption spectroscopic study on band-structure-type change in $\mathrm{CdTe} / \mathrm{CdS}$ core-shell quantum dots. IEEE J Quantum Electron 2011; 47: 1177-1184

42 Pan LY, Zhang YL, Wang HY, Liu H, Luo JS et al. Hierarchical self-assembly of CdTe quantum dots into hyperbranched nanobundles: suppression of biexciton Auger recombination. Nanoscale 2011; 3: 2882-2888.

43 Wang L, Wang HY, Wei HT, Zhang H, Chen QD et al. Unraveling charge separation and transport mechanism in aqueous-processed polymer/CdTe nanocrystal hybrid solar cells. Adv Energy Mater 2014; 4: 1301882.

44 Sykora M, Mangolini L, Schaller RD, Kortshagen U, Jurbergs D et al. Size-dependent intrinsic radiative decay rates of silicon nanocrystals at large confinement energies. Phys Rev Lett 2008; 100: 067401.

45 Kuntermann K, Cimpean C, Brehm G, Sauer G, Kryschi C et al. Femtosecond transient absorption spectroscopy of silanized silicon quantum dots. Phys Rev B 2008; 77: 115343.

46 Groenewegen V, Kuntermann V, Haarer D, Kunz M, Kryschi C. Excited-state relaxation dynamics of 3-vinylthiophene-terminated silicon quantum dots. J Phys Chem C2010; 114: 11693-11698.

47 Atkins TM, Thibert A, Larsen DS, Dey S, Browning ND et al. Femtosecond ligand/core dynamics of microwave-assisted synthesized silicon quantum dots in aqueous solution. J Am Chem Soc 2011; 133: 20664-20667.

48 Sommer A, Cimpean C, Kunz M, Oelsner C, Kupka HJ et al. Ultrafast excitation energy transfer in vinylpyridine terminated silicon quantum dots. J Phys Chem C 2011; 115: 22781-22788

49 Fuzell J, Thibert A, Atkins TM, Dasog M, Busby E et al. Red states versus blue states in colloidal silicon nanocrystals: exciton sequestration into low-density traps. J Phys Chem Lett 2013; 4: 3806-3812.

50 Kůsová K, Hapala P, Valenta J, Jelínek P, Cibulka $\mathrm{O}$ et al. Direct bandgap silicon: tensile-strained silicon nanocrystals. Adv Mater Interfaces 2014; 1: 1300042.

51 Kang ZH, Liu Y, Tsang CH, Ma DD, Fan X et al. Water-soluble silicon quantum dots with wavelength-tunable photoluminescence. Adv Mater 2009; 21: 661664.

52 Pi XD, Mangolini L, Campbell SA, Kortshagen U. Room-temperature atmospheric oxidation of Si nanocrystals after HF etching. Phys Rev B 2007; 75: 085423.

53 Dasog M, Yang Z, Regli S, Atkins TM, Faramus A et al. Chemical insight into the origin of red and blue photoluminescence arising from freestanding silicon nanocrystals. ACS Nano 2013; 7: 2676-2685.

54 Hannah DC, Yang JH, Kramer NJ, Schatz GC, Kortshagen UR et al. Ultrafast photoluminescence in quantum-confined silicon nanocrystals arises from an amprphous surface layer. ACS Photonics 2014; 1: 960-967.

55 Dasog M, De los Reyes GB, Titova LV, Hegmann FA, Veinot JG. Size vs surface: tuning the photoluminescence of freestanding silicon nanocrystals across the visible spectrum via surface groups. ACS Nano 2014; 8: 9636-9648.

$56 \mathrm{Kim}$ SW, Lee J, Sung JH, Seo DJ, Kim I et al. Two-dimensionally grown single-crystal silicon nanosheets with tunable visible-light emissions. ACS Nano 2014; 8: 65566562.

This work is licensed under a Creative Commons AttributionNonCommercial-ShareAlike 3.0 Unported License. The images or other third party material in this article are included in the article's Creative Commons license, unless indicated otherwise in the credit line; if the material is not included under the Creative Commons license, users will need to obtain permission from the license holder to reproduce the material. To view a copy of this license, visit http://creativecommons.org/licenses/by-nc-sa/3.0/ 